ARTículo

\title{
Los nuevos servicios locales de Educación Pública de Chile: desafíos del proceso de implementación inicial*
}

Sebastián Donoso-Díaz a

\section{Resumen}

Se analiza el proceso de instalación de los nuevos sistemas locales de Educación pública escolar, dirigido al fortalecimiento del sector, tras experimentar un importante deterioro en las últimas cuatro décadas. El proceso se desarrolla en un escenario internacional orientado en sentido contrario: privilegiar la privatización del sector. Para comprender el fenómeno se explicitan desde la perspectiva de los ciclos políticos, los trayectos históricos del país en las últimas décadas, las características del sistema municipal de Educación y el complejo avance de la implementación de los nuevos servicios locales en la actualidad. Se exponen las influencias técnicas de la ley, la simultaneidad de tareas y las tensiones políticas que genera una normativa basada en derechos sociales implementada por un gobierno con más interés en lo privado, en un escenario de incertezas que se busca precisar y reducir.

Palabras clave: Educación Pública. Institucionalidad subnacional. Implementación de reformas Educativas. Privatización de la Educación.

\section{Introducción}

Chile es un referente en materia de privatización de la Educación pública e incorporación del mercado a la Educación (FLORES, 2020). Si bien a partir de 1990 -en democracia- se impulsaron iniciativas destinadas a regular al sector privado y paralelamente apoyar al público de responsabilidad municipal, sus impactos fueron insuficientes para corregir las grandes desigualdades intra y

\footnotetext{
* Se agradece el apoyo financiero de la Agencia Nacional de Investigación y Desarrollo de Chile, Proyecto n 1190086, y también la colaboración de Daniel Reyes A.

a Universidad Talca, Talca, Maule, Chile.
} 
extra sistema escolar, dado que también -concomitantemente- en estos años se promovieron medidas como el copago (financiamiento compartido) en la Educación escolar subsidiada (1993) que consolidaron el mercado, como lo evidenciaron las demandas de los movimientos estudiantiles de 2006 y 2011, que convocaron a una profunda revisión de la Educación pública y del compromiso del Estado con el sector ${ }^{1}$ (BELLEI, 2015).

A partir de 2014, el gobierno promovió un conjunto de reformas dirigidas a fortalecer la Educación pública, inspiradas en "sacar a la Educación del mercado", entre las que destaca la ley que Crea el Nuevo Sistema de Educación Escolar Pública, planteando una nueva arquitectura (de organización y funcionamiento) mediante una institucionalidad ad hoc a nivel nacional que también implica un marco ideológico de la Educación ${ }^{2}$.

La reforma educativa de 1980 fue parte del diseño político del gobierno dictatorial dirigido a consolidar el modelo de Estado y sociedad neoliberal, que en 1981 instaló transformaciones estructurales decisivas, muchas de las cuales -con ajusteshan seguido vigentes, como la Constitución Política (que implanta el principio de subsidiariedad), la reforma del sistema de pensiones, la reforma educacional, y cambios en la regionalización del país, incluyendo la creación de municipios, buscando el protagonismo de tales autoridades como actores claves en el diseño de la nueva institucionalidad del Estado. De esa forma, se ha conformado un entramado razonablemente exitoso que ha permanecido -con adecuaciones menores- hasta hoy (VALDIVIA ORTIZ DE ZARATE, 2018).

Durante el año 2017, la Ley n 21.040 desplazaría la responsabilidad de la administración de la Educación pública desde los municipios al Nuevo Sistema de Educación Pública. Este nuevo sistema incorpora una unidad al Ministerio de Educación, la Dirección de Educación Pública (en adelante DEP), de la que dependerán los 70 Servicios Locales de Educación Pública (en adelante SLEP) que se generen según el diseño progresivo de su instalación y operación hasta el 2025, agrupando todos los establecimientos educacionales de las 345 unidades de gestión municipal en esta nueva institucionalidad.

El eje de la transformación es la nueva organización subnacional que desmunicipaliza el sistema escolar público. Esta modificación se propuso para mejorar la Educación ante la débil capacidad de los municipios para asumir

\footnotetext{
Se asume Educación Pública aquella financiada e implementada por entes del Estado central o subcentral.

2 El currículo escolar no es parte de esta reforma. Chile posee un currículo nacional obligatorio para la educación escolar.
} 
esta tarea y la excesiva intromisión política en la gestión educativa local de la autoridad municipal superior (Alcalde ${ }^{3}$ ) impactando negativamente en el desarrollo de la Educación pública en estas cuatro décadas (BELLEI, et al., 2018; RACZYNSKI; SALINAS, 2009).

La relevancia del estudio para el país, y también para la región latinoamericana, está dada por su envergadura y trascendencia, al ser una transformación que involucra a todos los establecimientos y niveles escolares (parvularia, básica y media) y sus unidades subnacionales de gestión, e incorporando una institucionalidad nacional, proceso muy desafiante (CONCHA; GARCÍAHUIDOBRO, 2020). Eso, especialmente porque este cambio conlleva transformar el elemento vertebrador y estratégico del sistema educativo de las últimas cuatro décadas: la municipalización de la Educación, sumando un elemento adicional, cuasi-inédito: fortalecer la institucionalidad pública, cuando en la actualidad muchos países piensan en reforzar el sector privado paralelamente con reducir el sistema público (ANDERSON, 2018). De esa manera, se impacta finalmente en el cambio del diseño político del Estado impuesto en dictadura, que es un factor interesante de considerar.

\section{El problema en estudio y su sustento}

El análisis de esta reforma en curso implica abordar uno de los procesos de cambio más complicados e inéditos que está experimentando el Estado chileno en Educación y que se extenderá posiblemente por una década más. Por ende, el trabajo pretende relevar y analizar las condiciones estructurales y políticas de la fase inicial de la implementación del nuevo sistema de Educación pública (en adelante NEP), extrayendo algunas Enseñanzas de este proceso, en especial respecto de los SLEP, que sustituyen a los municipios bajo nuevas funciones.

Desde la teoría, siguiendo a Mainardes (2006), el estudio corresponde al tercer nivel de análisis de la investigación en política educativa "que se centra en las estructuras: "políticas de la educación" (p. 154). En razón de ello, el eje observado no son directamente los movimientos estudiantiles, ni las teorías que analizan los sistemas educacionales contemporáneos, o las políticas públicas llevadas a cabo en las últimas décadas, sino en la configuración del escenario en el cual estos componentes interactúan, deteniéndonos en su trasfondo para el sistema educacional, asumiendo, "las políticas públicas como producto del proceso político” (SECCHI, 2014, p. 23).

\footnotetext{
3 Por ello se habla de "desalcalización" de la educación, pues es un factor determinante del cambio, no es solo un traslado de dependencia, sino un modelo de gestión diferente.
} 
Se entiende que estos últimos no son lineales, ni se desarrollan en etapas secuenciadas o excluyentes, y que generan interacciones multidireccionales entre el nivel macro, meso y micro, originando diversas dinámicas. La aproximación al fenómeno se realiza desde el enfoque de ciclos políticos, entendidos como ideas aglutinadoras que comparten conceptos y prácticas distintivas histórico-sociales con procesos que varían en el tiempo y en contextos (DESOUZA; SECCHI, 2015).

La formulación de políticas traduce los propósitos de los gobiernos en programas y acciones que buscan generar resultados o cambios. En este marco, el Estado es un ámbito de tensión y disputa donde se conjugan intereses de distintos sectores. Las políticas dan cuenta del ajuste y priorización de las acciones, de la negociación entre actores y, siguiendo a Weber, implican visualizar el tema del poder: la capacidad de imponer a otro la propia voluntad (SALVAT, 2014).

El análisis de la NEP es complementado con el enfoque de Ciclo de Políticas Educativas de Bowe, Ball y Gold (1992), destacando la naturaleza compleja del fenómeno, y diseñando un modelo analítico de implementación, entendiendo que en la práctica los actores no le siguen literalmente. Ball (2015) señala que el ciclo implica identificar tensiones y disputas, como también la autonomía y el poder de los actores sociales en y fuera del Estado. Además, permite analizar las políticas sociales en el contexto, entendiendo que toda política conlleva consideraciones teóricas y operacionales sobre la gestión del Estado. Complementando esta perspectiva, Mainardes (2006) señala que Ball "rechaza los modelos de política educacional que separan las fases de formulación e implementación porque ignoran las disputas sobre la política" (2006, p. 49). Además, al situar el análisis en el contexto, precisando su fase inicial, incluye la dinámica de la adecuación local y el rol de estos actores (SABATIER, 1986, p. 37), implementando las políticas públicas como búsqueda de soluciones a los problemas en el lugar/momento más propio donde emergen. Se entiende que toda política es adaptable a las variaciones locales y a los agentes más idóneos que serían "aquellos que la implementan regularmente" (SECCHI, 2014, p. 61).

Si bien es temprano para una evaluación de los SLEP, se atenderá a la coherencia entre la intención propuesta y el grado en que se ha desarrollado (WOLLMANN, 2007). Se adopta un enfoque ex-post, dirigido al desempeño de la política pública, "con la intención de conocer su estado y el grado en que se reduce el problema que la generó" (SECCHI, 2014, p. 63). Este caso incluye el momento histórico del ciclo en desarrollo, que corresponde a su primera fase de implementación ${ }^{4}$.

\footnotetext{
4 El diseño de la NEP señala que el 2021 (funcionando 11 de los 70 SLEP), el Presidente(a) de la República, previa consulta al Consejo de Evaluación (31.03.2021), puede extender la instalación otros 5 años (2030).
} 


\section{Consideraciones metodológicas}

Desde la perspectiva temporal, se busca relevar las condiciones estructurales y políticas de la fase de formulación e implementación de los SLEP como componente fundamental de la NEP. Se consideran diversos ciclos políticos hasta llegar a su instalación (2018-2019), que incluye el comienzo del segundo semestre del segundo año de operación (Julio de 2019). Lo anterior, dado que desde mediados de octubre de 2019, producto del "estallido social" ocurrido en Chile ${ }^{5}$, se alteró radicalmente el año escolar, adoptándose medidas extraordinarias para su finalización, situación que se profundiza el 2020 por el impacto del Covid-19, en el desarrollo de las actividades escolares.

En razón de ello, el análisis se centró en la operación de la DEP y esencialmente de los primeros cuatro SLEP en régimen (Barrancas, Puerto Cordillera, Huasco y Costa Araucanía). No se considera la instalación y operación de los tres nuevos que debían iniciar sus operaciones en el 2019, por las razones indicadas. Las fuentes de información son: documentos oficiales del Ministerio, de la NEP, SLEP y otros entes públicos. Además de eso, información de prensa, estudios producidos y entrevistas semiestructuradas realizadas a informantes claves: cuatro directivos de los SLEP, dos integrantes de Comité Directivo local, y dos ex directivos de SLEP. Las limitaciones del estudio se refieren al hecho de que no fue posible exponer todos los hechos de manera cronológica desde la concepción de la política hasta la actualidad.

\section{Elementos claves del Nuevo Sistema de Educación Pública ${ }^{6}$}

La NEP es una institucionalidad pública especializada en gestión educacional, integrada por el Ministerio de Educación, la Dirección de Educación Pública, los Servicios Locales de Educación Pública y los establecimientos educacionales. El Estado provee Educación pública, gratuita ${ }^{7}$ y de calidad, mediante los establecimientos educacionales de su propiedad y administración integrados en los SLEP, garantizando el derecho a Educación dispuesto en la Constitución. A la DEP le corresponde la conducción estratégica y coordinación

\footnotetext{
5 La cronología inicial de los sucesos puede verse en https://www.dw.com/es/la-cronolog\%C3\%ADa-delestallido-social-de-chile/a-51407726

6 Texto resumido de "https://www.bcn.cl/leyfacil/recurso/nuevo-sistema-de-educacion-publica

7 La educación pública chilena, post reforma de 1980, definió un sistema de financiamiento de subsidio a la demanda de valor único (vigente sin cambios mayores hasta el 2008) aportado por el Estado al empresario (privado o publico). Al instalarse el copago, muchos establecimiento privados con subsidio público pudieron exigir directamente a los padres un pago adicional; por esta razón, la gratuidad, siendo consustancial a la educación pública, en Chile no lo fue plenamente sino en forma gradual desde el año 2014.
} 
del Sistema, es parte del Ministerio de Educación, y de ella dependen los SLEP. Estratégicamente la DEP debe formular la Estrategia Nacional de Educación Pública ${ }^{8}$.

Los SLEP serán dirigidos por un equipo técnico y además colegiadamente por un Comité Directivo Local, con representantes de los padres, municipalidades y gobierno regional. Además, existen los Consejos Locales de Educación Pública, con participación de docentes, asistentes de la Educación, estudiantes, padres y apoderados e instituciones de Educación Superior. Esta visión opera desde una perspectiva que implica mayor participación, trabajo en red y una forma de relacionar la Educación en la comunidad, que difiere de la administración municipal precedente.

Los SLEP son órganos públicos funcionales y territorialmente descentralizados, con personalidad jurídica y patrimonio propios. Su objeto es proveer el servicio educacional en los niveles y modalidades que corresponda, velando por la calidad, mejora continua y equidad del servicio. Los SEP deben entregar soporte técnico-pedagógico y apoyo a la gestión de los establecimientos, considerando sus proyectos educativos institucionales, la autonomía y necesidades de cada comunidad educativa, atendiendo a las características de sus estudiantes y del territorio. Cada SLEP deberá elaborar un Plan Estratégico Local para el desarrollo de la Educación en su área geográfica. Los establecimientos educacionales son la unidad básica del Sistema. Sus comunidades educativas la integran estudiantes, padres/apoderados, profesionales y asistentes de la Educación, y docentes directivos. Dichos establecimientos cuentan con autonomía para la definición y desarrollo de sus proyectos educativos ${ }^{9}$.

En el caso de los establecimientos dependientes de los SLEP, sus directores deben elaborarle, en conjunto con la comunidad educativa, y proponer al Director Ejecutivo del SLEP el Plan de Mejoramiento Educativo.

\section{El camino hacia la desmunicipalización de la Educación Pública}

La municipalización fue un punto de inflexión de la política educativa que implicó cambios notables en la organización del sistema escolar, traspasando la infraestructura, equipamiento, personal docente y administrativo desde el

\footnotetext{
8 La cual debe ser aprobada por el Consejo Nacional de Educación.

9 Muchas de estas atribuciones ya estaban en la Ley General de Educación (2009); el hecho que se señalen es un tema legal, pero pedagógicamente en esta dimensión no hay grandes diferencias.
} 
Ministerio de Educación a los municipios, y financieramente incorporando el subsidio por alumno, propio del mercado, manteniéndose como criterio dominante del financiamiento de las escuelas públicas, incluidas las de los SLEP. Conceptualmente, la municipalización quebrantó los principios fundantes de la provisión de Educación pública promovida por el Estado en el siglo XX: cumplir un rol decisivo en la organización del sistema escolar, ser protagónico en su provisión (incluyendo el financiamiento), otorgando centralidad a la Educación pública (gratuita) por sobre la privada. El primer principio se cambiaría por la subsidiariedad del Estado, donde la Educación pública cumple su papel cuando los privados no tienen interés en asumirle; y el segundo, desplazaría la atención hacia la privatización del sistema, generando las condiciones para ser provista mayoritariamente por privados (JOFRÉ, 1988; NÚÑEZ PRIETO, 1984, 2015) ${ }^{10}$.

El análisis del eje “municipalización/desmunicipalización”, como institucionalización del sistema escolar público, contribuye a comprender los alcances del devenir entre los ciclos políticos, a saber: el de la reforma dictatorial (1981-1990) que cimenta y consolida el modelo con instrumentos de mercado (JOFRÉ, 1988), bajo la modalidad de cuasi-mercado (JOIKO, 2012); el que culmina con la dictación del marco normativo protector del modelo, la Ley Orgánica Constitucional de Enseñanza (Loce) y la de Bases del Ministerio de Educación, ambas promulgadas los días finales del gobierno dictatorial, retardando sus transformaciones hasta las demandas de cambio del movimiento estudiantil de 2006.

El ciclo democrático inicial (1990-2006) realizó adecuaciones al sistema imperante, lo que significó dictar el Estatuto Docente (1991), subir el valor de los subsidios escolares, impulsar la Reforma Curricular de 1995 en adelante, y otras medidas dirigidas a regular el destino de los recursos públicos entregados a los sostenedores ${ }^{11}$. Además, enfatizar la preocupación por el mejoramiento de los indicadores escolares de eficiencia interna y externa, sin cambiar la arquitectura institucional del sistema (CONSEJO ASESOR PRESIDENCIAL..., 2006). El incremento de la eficiencia en esos aspectos también validaría el mercado, no solo por la introducción del copago (1993), sino por haber fortalecido la competencia como camino a seguir igualmente por los municipios (ORGANIZACIÓN PARA LA COOPERACIÓN Y EL DESARROLLO ECONÓMICO, 2004). No obstante, el sistema público

\footnotetext{
${ }^{10}$ Pese a ello, el Estado financia en forma relevante el $90 \%$ de la matricula escolar.

11 Se define como sostenedor al responsable/propietario de la gestión educacional de un establecimiento, sea un empresario privado o público (Alcalde o su representante).
} 
acusaba problemas endémicos de organización y financiamiento (GONZÁLEZ, 2003) que repercutían en sus resultados de aprendizajes, dando origen a las primeras críticas sobre lo obrado en democracia (BELLEI; CONTRERAS; VALENZUELA, 2009) y la posibilidad de revisar la institucionalidad pública subnacional (POLITEIA, 2008).

El ciclo siguiente (2006-2011) marca el avance del desgaste de las propuestas educativas de los gobiernos, alineadas con las manifestaciones sociales de las tensiones más significativas de la Educación pública: la búsqueda de soluciones, y la persistencia de los efectos educativos negativos, a saber: su baja incidencia en reversar las grandes desigualdades educacionales de la sociedad, la alta segmentación socio-territorial y los problemas históricos de recursos financieros y calidad de la Enseñanza. Los avances en este plano permitieron sustituir la Loce, por la Ley General de Educación, que implicaría un avance en organizar y regular el sistema escolar, aunque sería una respuesta parcial ante las demandas más sustantivas, por cuanto no abordaría la municipalización de la Educación, el financiamiento vía subsidio a la demanda y las posibilidades de lucro del sector privado, cuestionados por el movimiento estudiantil de 2011, que propuso aspiraciones de cambios más profundos, que implicaban la institucionalidad educativa subnacional (DONOSO, 2013), problemas reconocidos por los responsables de las reformas educativas iniciales en democracia,

Dos efectos no buscados por las políticas de la Concertación y de gran relevancia política desde las protestas de 2006, renovadas a escala expandida por el estudiantado universitario en 2011, son la segregación social del sistema y la disminución de la matrícula de la educación municipal. Las dos dinámicas están interrelacionadas y son inseparables de la regulación de mercado del sistema en su conjunto, establecida en la década de los Ochenta y que las políticas del período 1990-2010, no neutralizaron (COX, 2012, p. 32).

En el ciclo siguiente (2012-2017) destacan la agudización de los conflictos públicos del sistema escolar; y el debate y diseño de nuevos caminos de corrección de los principales nudos críticos, esencialmente los institucionales, referidos a la desmunicipalización escolar y gratuidad de la Educación Superior, materias instaladas en la política nacional, e inevitables en la agenda de la campaña presidencial de 2013, que derivaron -posteriormente- en distintas leyes, entre las que destaca la del nuevo sistema de Educación pública.El actual ciclo, iniciado el 2018, consigna el proceso de implementación de la NEP, que debiese extenderse al 2025, dando cuenta - entre otras materias-de las dificultades entre el diseño de 
una reforma inspirada en el derecho social, pero implementada por un gobierno identificado con la privatización del sistema público.

En esta trayectoria, los puntos de inflexión claves del eje político "hacia la desmunicipalización", han sido los movimientos estudiantiles mencionados, que situaron en el debate nacional los problemas de la Educación pública y los efectos sociales de la institucionalidad local educativa generada en dictadura. El movimiento de 2006 posibilitó por primera vez - posdictadura- discutir públicamente algunos de los problemas estructurales de la Educación, destacando la necesidad de realizar transformaciones institucionales mayores más que adecuaciones al sistema (BELLEI et al., 2018). La extensión de esta crisis más allá de una década -2006 en adelante-, expone la complejidad del contexto que media entre las visiones pro-adecuación del modelo, y las transformaciones mayores de sus principios y estructuras de operación (DONOSO-DÍAZ et al., 2015).

En referencia a esos ejes, se organizan las principales demandas del movimiento de 2011: fin al lucro de las instituciones educativas (de todos los niveles), cambio del sistema de financiamiento de la Educación -buscando reconocerle como derecho social-y en la estructura del sistema educativo público, expresados en la consigna: "educación pública, gratuita y de calidad" (GARCÍA-HUIDOBRO; FERRADA; GIL, 2014). En función de estos argumentos se articulan los debates, incorporando conceptos como una nueva organización del Estado y de la Educación, relacionada con las demandas ciudadanas, con nuevas visiones ante los esquemas convencionales de organización y acción de los actores políticos y los movimientos sociales (GARRETÓN, 2016; VALDIVIA ORTIZ DE ZATATE, 2018),

Más allá de las correcciones para "neutralizar" los efectos del mercado en Educación ocurridas en democracia, las críticas a sus impactos negativos derivaron en presiones sobre el diseño político bajo el cual ha funcionado el Estado y la sociedad (MAYOL, 2012; MOULIAN, 2002), incidiendo de manera decisiva en los temas estratégicos de la agenda de la campaña presidencial de 2013 de las coaliciones dominantes. Por ende, el nuevo gobierno -Bachelet 2014/2017intentará conducir la discusión hacia el fin lucro en la Educación (CORVALAN; GARCÍA-HUIDOBRO, 2016), plasmando en este marco varias leyes, entre ellas, la del nuevo sistema de Educación pública.

Con antelación, el informe de la OCDE sobre la Educación chilena (OCDE, 2004), alertaba sobre la gestión municipal señalando "las municipalidades son muy heterogéneas, $[\ldots]$ difieren en su capacidad para administrar recursos 
educacionales. Difieren en su interés por la educación y en la capacidad para abordar problemas y soluciones" (OCDE, 2004, p. 280).

Dos años después, el informe del Consejo Asesor ${ }^{12}$ (2006) señalaba que había acuerdo -no consensuado- en que "el esquema institucional ha mostrado limitaciones vacíos e inconsistencias" (CONSEJO ASESOR PRESIDENCIAL..., 2006, p. 111).

El Consejo concuerda que el desempeño del actual sistema de administración municipal de la educación es negativo. Hay acuerdo en las condiciones que explican las falencias del sistema municipal, un financiamiento insuficiente para las obligaciones que poseen, disolución de responsabilidades entre el Ministerio y los municipios, escasa transferencia de competencias a las escuelas, relación desigual entre el sector municipal y el particular subvencionado. Algunos consejeros señalan que también influye el tamaño y sus capacidades de gestión entre ellos (CONSEJO ASESOR PRESIDENCIAL..., 2006, p. 115).

Finalmente, se enfatizaba que la estructura municipal, por el poder asimétrico del Alcalde y una institucionalidad débil, focalizada en muchas tareas, requerían otro marco institucional para la Educación pública, imponiéndose la desalcaldización (BELLEI; CONTRERAS; VALENZUELA, 2009; LARRAÑAGA; PEIRANO; FALCK, 2009).

En síntesis, en la discusión pública se impuso una visión dominante que visualizaba que para la mejora significativamente de la Educación pública era imprescindible crear una nueva institucionalidad que reemplazara la gestión de los municipios y los alcaldes, y consolidara una institucionalidad autónoma, especializada y sustentable en el plano subnacional.

\section{Análisis del proceso inicial de Instalación de los SLEP}

El análisis de instalación de los SLEP muestra situaciones cuya sumatoria generan un escenario de importancia, previsible en parte, considerando las experiencias de otros casos, en términos de escala de tareas (OCDE, 2015) y algunas incógnitas sobre su proyección para el mediano plazo.

\footnotetext{
${ }^{12}$ Este informe es una pieza clave que contiene visiones y propuestas que fueron desarrolladas en los años siguientes, teniendo un valor insustituible en el estudio del tema.
} 
Junto a lo inédito del proceso de fortalecimiento del sistema de Educación pública, se adiciona la complejidad de acometer una labor de esta envergadura en un periodo tan acotado de tiempo (tres meses entre la aprobación de la ley y la entrada en operación de los primeros dos SLEP), cuya fundación se realiza sobre un sistema que ha funcionado durante cuatro décadas, con falencias importantes en gestión. De manera que el reto es que esta transformación no incida negativamente en los resultados escolares, lo que en el corto plazo es complejo, aunque a mediano plazo serán los aprendizajes de sus estudiantes un indicador clave para el éxito. No obstante, hay desafíos urgentes que atender para mejorar el proceso en este plano.

\subsection{Principales puntos críticos del proceso de instalación}

La NEP implica una nueva organización e institucionalidad que se adosa a un Ministerio saturado de unidades, traduciéndose en mayor complejidad estructural y operacional, porque también la DEP debe apoyar a los SLEP, estando todos en instalación; como señala un Directivo Local:

hay un tema de recentralización de la gestión que es un obstáculo inicial, dado que la cultura Ministerial es esperar las instrucciones del 'centro', práctica que inmoviliza la gestión (...) además porque los cambios de autoridades de la DEP y SLEP, y su lenta renovación dieron mayor inmovilismo (Directivo Local).

Si bien la instalación de los SLEP se realizó tensionada por autoridades que duraban un año y que no habían sido nombradas por el nuevo gobierno (URIBE; VALENZUELA; ANDERSON, 2019, p. 165), y que los cambios de éstas (DEP y SLEP) retrasaron casi un semestre los nuevos cargos en propiedad ${ }^{13}$, se logró que el sistema funcionara y ello es importante. Se hizo sobre la base de mucho esfuerzo de quienes estaban en estas unidades, producto de aprendizajes, errores, y vacíos que hubo que solucionar rápidamente.

los quehaceres administrativos consumieron mucho trabajo, tensionándose el sistema con tareas sincrónicas que implicaron, por ejemplo: manuales de procedimientos provisionales, personal nuevo -provenientes del mundo privado- que no conocía los procesos del sector público, con capacitaciones muy recientes, plataformas de

\footnotetext{
${ }^{13}$ Cumplido el primer año, todos los cargos directivos superiores fueron removidos y reemplazados por los del nuevo Gobierno, con carácter interino. Ese proceso además continuó hasta mediados del 2019, con la rotación de esos cargos (incluyendo Director de la DEP), lo que derivó en que el sistema operara en su mínimo (SEGOVIA, 2019).
} 
trabajo en instalación, y mucho menos personal que el necesario para asumir las tareas (ex directivo SLEP).

La operación de los SLEP enfrenta otros desafíos de relevancia: (i) integrar equipos directivos y docentes con distintas prácticas, nuevas tareas e incluso salarios y beneficios laborales disímiles entre los municipios, lo que conlleva una adecuación superior a lo previsto; (ii) cambiar los mecanismos de operación y de comunicaciones de los equipos técnicos en territorios más grandes, con profesionales en instalación en todos los niveles y, con normativas en desarrollo y adecuación; (iii) cambiar el plan de integración de los municipios a los SLEP según sus problemas financieros previos (URIBE; VALENZUELA; ANDERSON, 2019).

Casi todos creíamos necesaria la NEP, el problema fue que la instalación se hizo con escasa comunicación a todos, no solo a profesores y familias, sino a los profesionales y directivos; y además teníamos dotaciones inconsistentes con las competencias requeridas de los diversos cargos, lo que no ayudaba (Directivo SLEP).

En el equipo técnico de los SLEP se nombraron profesionales que provenían del sector privado que no tenían experiencia en gestión pública, situación inadecuada en momentos donde hay poco tiempo para aprender acerca de licitaciones, adquisiciones, pagos y sus plazos, y fundamentalmente la complejidad de los pagos de remuneraciones, que son cruciales de impulsar en determinados plazos y períodos anuales, y que han mostrado retrasos relevantes imputables a inexperiencia, dilatando la instalación y precarizando sus resultados.

Hubo muchos retrasos por problemas de escases de personal, a veces por falta de dominio técnico de los procesos administrativos, y por la poca información que todos teníamos, y porque tampoco en la DEP resolvían del todo algunas consultas (Directivo local).

Adicionalmente, durante gran parte del tiempo considerado en el estudio, los equipos técnicos de los SLEP han operado con menos del $40 \%$ de los funcionarios previstos, lo que implica retrasos en materia de tareas regulares y sustantivas que debiesen redundar en inconvenientes operativos que impactan los resultados de los nuevos servicios (CERRO, 2019, p. 49 y ss).

El primer año lo destinaos a la instalación y operación fundamental, que funcionara el sistema de remuneraciones, de compras, de organización de los establecientes y la matrícula. Lo técnico 
pedagógico no pudimos verlo sino en momentos puntuales, atender un territorio mucho más grande, con menos personal impidió cumplir mejor esa tarea (Directivo SLEP).

Otro punto crítico se refiere al gasto público efectivo, a julio de 2019, según datos de la Dirección de Presupuestos, la DEP había ejecutado el 21\% de su presupuesto, cifra muy por debajo de lo esperado a esa fecha, teniendo en cuenta que la ejecución del año anterior fue de $97 \%$. En el caso de los SLEP, la situación es similar en términos de tendencia, aunque la diferencia es menor. El año 2018 se ejecutó el 85\% del presupuesto y el 2019 el 51\% (DONOSO-DIAZ et al., 2019). Lo señalado es coherente con la complejidad de impulsar un proceso de esta envergadura, la inexperiencia de liderar cambios contra-cíclicos (reorganizar lo público desde lo público), y las tensiones derivadas de los cambios de administración del gobierno nacional ocurridos en esta fase.

Hay cuestiones relevantes en materia de gestión que requieren de otro tipo de atención para mejorar este proceso. Primero, poner mayor dedicación al accionar de los municipios en el periodo previo al traspaso, inicialmente en lo financiero:

que los municipios provean antecedentes financieros y contables rigurosos, que incluyan personal, infraestructura y otras partidas indispensables para su proyección (Directivos SLEP).

En algunos DAEM ${ }^{14}$ se incrementó la dotación de personal y se mejoraron las condiciones contractuales, previo al traspaso al SLEP, incluyendo el traslado de parte de su dotación a las escuelas, generando un incremento en el gasto permanente, que ha desestabilizado el presupuesto (Directivos SLEP).

Segundo, en el trabajo previo entre municipios y la DEP; la colaboración en este proceso entre estas unidades resulta determinante para la fluidez de los procesos:

En el territorio había trabajo previo y conjunto de los municipios y una buena relación con el MINEDUC, ello fue importante para la pronta instalación, el traspaso de recursos y de los equipos humanos, y en especial para resolver imprevistos. Los municipios entendían que la NEP beneficia a su población (Directivo local).

${ }^{14}$ Departamento de Administración de Educación Municipal. 
El problema fue que no se hizo un plan de anticipación profundo para trabajar con todas las comunas involucradas en el SLEP, ello habría reducido algunos problemas de acceso a información que tuvimos (Directivo SLEP).

Otro elemento importante es la percepción de la capacidad de los directivos del SLEP y su validación en el medio/contexto en el cual se instala, tanto intra equipo técnico del SLEP, como respecto de los directivos de establecimientos y los docentes:

hay algunos integrantes del equipo técnico que creen que "hacen un favor" no cerrando escuelas pequeñas, eso ha sido negativo para su aceptación por quienes estamos en la educación pública por tiempo, docentes y directivos (Directivo Local)

Los directivos SLEP que tienen prestigio y respeto por su trayectoria y desempeño profesional en el contexto local son creíbles, eso ayuda a liderar este proceso (Directivo SLEP).

Lo señalado es altamente relevante en lo operacional, que es un campo donde una reforma se lleva a cabo. Aunque no todo se reduce a cuestiones operativas, hay un tema de fondo, el indispensable cambio de mentalidad que debe ocurrir en un plazo intermedio:

La NEP, por sobre las dificultades que ha mostrado [...] debe aportar con una forma diferente de pensar, organizar y practicar la educación en escuelas y en lo local. [...] Si no resulta pronto -posiblementeserá una buena reforma, pero no "la gran reforma" que potencie a la educación pública hacia el futuro (Directivo Local).

\subsection{Debate final}

El análisis de la estructura política de la reforma educativa, en el contexto/escenario en el que se instala la NEP, confirma su complejidad dada la insuficiente sintonía que ha existido entre sus fundamentos y la implementación en una intencionalidad opuesta a la prevalencia del mundo privado. Si bien ello era previsible, es interesante que en la literatura sobre reformas educativas (GILLIES, 2010; OCDE, 2015) varias advertencias podrían haberse considerado para aumentar el grado de éxito de esta etapa. 
Dicha "asintonía" agrega tensiones a los actores involucrados en el proceso. Aunque toda política posee un grado de imprevisibilidad, en este caso aumenta por la dificultad de impulsar una reforma de esta envergadura en un breve plazo y con componentes divergentes en el plano contextual.

Chile está definiendo un ciclo con ajustes entre los tiempos políticos y los técnicos, dado que no se comparten las principales ideas aglutinadores, ni conceptos unívocamente asumidos; se trata de un proceso en el que se están instalado recientemente prácticas distintivas histórico-sociales respecto de gestión local y participación incipientes y que requieren de apoyo y sustentabilidad en el tiempo para estimular cambios en la forma de pensar y luego en el actuar, que son el desafío central para consolidar una reforma que requiere de un cambio de mentalidad que conlleve un hacer diferente.

Las transformaciones de fondo del sistema educativo público se relacionan con "sacar al mercado de la lógica del sistema educacional", cambio teórico definitorio, generando procesos sustentables en el tiempo, para provocar efectos positivos en las prácticas de colaboración entre establecimientos, de nuevos formatos de planificación participativa, como también de no selección de estudiantes (fin al lucro), que implican instalar un nuevo Estado local en Educación, una tarea política muy relevante para rediseñar la herencia negativa dejada por la dictadura en Educación.

Los actores consultados tienen claro que la NEP es una experiencia valiosa y necesaria, reconocen los problemas de instalación y conducción con el Ministerio y también de relación con los Municipios, y el valor de la significación del momento que se vive para el éxito de la reforma. Además, dan pistas claves sobre la importancia de preparar el camino institucional y también con los actores internos y externos del sistema. Se podría argumentar que nada de esto es nuevo. Lo interesante es preguntarnos ¿Por qué en su diseño original no se contemplan en detalle estos aspectos si se conocía su relevancia? Una respuesta es que también esta manera de generar reformas ha entrado a su ciclo final; hay indicios de nuevos caminos, mucho más potentes de gestión "de abajo-hacia arriba".

Si bien la reforma en su fase inicial se ha instalado, pese a los contratiempos, hay cuestiones sustantivas que podrían atenderse, como la recentralización que implica la gestión de la DEP, cuando paralelamente la descentralización del país avanza, lo que provoca "ruidos" de gestión que deben anticiparse, dado que los 
gobiernos subnacionales tendrán mayor poder y atribuciones y la institucionalidad de los SLEP no dialoga con ello.

No obstante, la indivisibilidad pública de esta temática, ayudada por la Pandemia, no facilita que exista mayor atención sobre su devenir, siendo factible que las otrora incipientes brechas que no se han cerrado en materia de gestión, de apoyo del Mineduc, y de otros elementos expuestos, reduzcan el andar de esta reforma, llevándonos a un escenario aún más complejo. La coexistencia hasta el año 2030 de dos sistemas de Educación pública con marcos operacionales muy distintos, finalmente podría reducir el ejercicio del derecho a Educación -esencialmentede los más vulnerables. 


\title{
The new local services of Public Education in Chile: Challenges of the initial implementation process
}

\begin{abstract}
The process of installing the new local public school Education systems is analyzed; this process aimed at strengthening the sector, after experiencing a significant deterioration in the last four decades. The process takes place in an international scenario oriented in the opposite direction: favoring the privatization of the sector. In order to understand the phenomenon, the historical trajectories of the country in recent decades, the characteristics of the municipal Education system and the complex progress of the implementation of new local services today are made explicit from the perspective of political cycles. The technical influences of the law, the simultaneity of tasks and the political tensions generated by a regulation based on social rights implemented by a government with more interest in the private, are exposed, in a scenario of uncertainty that is sought to be specified and reduced.
\end{abstract}

Keywords: Public Education. Subnational Institutionality. Implementation of Educational Reforms. Privatization of Education.

\section{Os novos serviços locais da Educação Pública no Chile: desafios do processo de implementação inicial}

\section{Resumo}

É analisado o processo de instalação de novos sistemas de Ensino Público Municipal, visando ao fortalecimento do setor, após ter experimentado uma significativa deterioração nas últimas quatro décadas. O processo se dá em um cenário internacional voltado na direção oposta: privilegiar a privatização do setor. Para a compreensão do fenômeno, explicam-se as trajetórias históricas do país nas últimas décadas, as características do sistema municipal de ensino e o complexo andamento da implantação de novos serviços locais sob a ótica dos ciclos políticos atuais. São expostas as influências técnicas da lei, a simultaneidade de tarefas e as tensões políticas geradas por uma regulação baseada nos direitos sociais implementada por um governo com mais interesse na questão privada, em um cenário de incertezas que busca especificar e reduzir.

Palavras-chave: Educação Pública. Institucionalidade Subnacional. Implementação de Reformas Educacionais. Privatização da Educação. 


\section{Referencias}

ANDERSON, S. Servicios locales de educación y el mejoramiento escolar. 24 ago. 2016. Dinponible en: https://www.lidereseducativos.cl/recursos/videostephen-anderson-servicios-locales-de-educacion-y-el-mejoramiento-escolar/. Acceso en 15 abr. 2018.

BALL, S. What is policy? 21 years later: reflections on the possibilities of policy research. Discourse: Studies in the Cultural Politics of Education, [s. 1.]. v. 36, n. 3, p. 306-313, 2015. http://dx.doi.org/10.1080/01596306.2015.1015279

BELLEI, C. El gran experimento: mercado y privatización de la educación chilena. Santiago: LOM. 2015.

BELLEI, C. et al. La nueva educación pública: contexto, contenidos y perspectivas de la desmunicipalización, Santiago: Universidad de Chile, 2018.

BELLEI, C.; CONTRERAS, D.; VALENZUELA, J. P. Fortalecer la educación pública: un desafío de interés nacional. In: BELLEI, C; CONTRERAS, D; VALENZUELA, J. P. (eds.). Ecos de la revolución pingüina: avances, debates y silencios de la reforma educacional. Santiago, Universidad de Chile, UNICEF, 2009. p. 225-256.

BOWE, R.; BALL, S.; GOLD, A. Reforming education and changing schools: case studies in policy sociology. London: Routledge. 1992.

CERRO, C. El nuevo sistema de educación pública chilena: fundamentos, elementos para su puesta en marcha y situación actual. Talca: IIDE-Universidad de Talca, 2019.

CONCHA, C.; GARCÍA-HUIDOBRO, J. E. La educación pública, una construcción histórica. In: CORVERA, M. T.; MUÑOZ, G. (eds.). Horizontes y propuestas para transformar el sistema educativo chileno. Santiago: Ediciones BCN, 2020. p. 278-299.

CONSEJO ASESOR PRESIDENCIAL PARA LA CALIDAD DE LA EDUCACIÓN. Informe final. Santiago: Presidencia de la República, 2006.

CORVALAN, J; GARCÍA-HUIDOBRO, J. E. Educación y mercado: el caso chileno. In: CORVALAN, J; CARRASCO, A; GARCÍA-HUIDOBRO, J.E. Mercado escolar y oportunidad educacional: libertad, diversidad. Igualdad. CEPPE., Santiago, 2016. p. 17 -56. 
COX, C. Política y políticas educacionales en Chile 1990-2020. In: Revista Uruguaya de Ciencias Politicas, Montevideo, v. 21, n. 1, p. 9-28, ene. 2012.

DESOUZA, G; SECCHI, L. Extinção de políticas públicas: síntese teórica sobre a fase esquecida do policy cycle. Cadernos Gestão Pública e Cidadania, São Paulo, v. 20, n. 66, p. 75-93, jan./jun. 2015. http://dx.doi.org/10.12660/cgpc.v20n66.39619

DONOSO, S. Dynamics of change in Chile: explaning the emergence of the 2006 pingüino movement. Journal of Latin American Studies, v. 45, n. 1, p. 1-9, feb. 2013. https://doi.org/10.1017/S0022216X12001228

DONOSO-DÍAZ, S., et al. Nudos críticos del sistema escolar chileno, hipótesis explicativas y propuestas para una nueva arquitectura del sector público. Ensaio. Avaliaçao e Politicas Publicas em Educação, Rio de Janeiro, v. 23, n. 86, p. 37-71, jan./mar. 2015. https://doi.org/10.1590/S0104-40362015000100002

DONOSO-DÍAZ, S. et al. La ejecución del gasto del nuevo sistema de educación escolar pública en Chile 2018-2019: disonancias entre la letra y espíritu de la reforma. Propuesta Educativa, v. 28, n. 52, p. 41, 56, nov. 2019.

FLORES, C. De la cobertura a la calidad de la educación. In: FALABELLA, A.; GARCÍA-HUIDOBRO, J. E. (eds.). A 100 años de la ley de instrucción primaria obligatoria: la educación chilena en el pasado, presente y futuro. Santiago: UAH, 2020. p. 198-206.

GARCÍA-HUIDOBRO, J. E.; FERRADA, R.; GIL, M. La relación educación-sociedad en el discurso político-educativo de los gobiernos de la Concertación (1990-2009). Estudios Pedagógicos (Valdivia), v. 40, n. 1, p. 113-131, 2014. https://doi.org/10.4067/S0718-07052014000100007

GARRETÓN, M. A. La ruptura entre política y sociedad: una introducción. In: GARRETÓN, M. A. (comp.). La gran ruptura, institucionalidad política y actores sociales en Chile del siglo XXI. Santiago: LOM, 2016. p. 11-20.

GILLIES, J. The power of persistence: education system reform and aid effectiveness: Case studies in long-term education reform. [S. 1.]: USAID, 2010.

GONZÁLEZ, P. Estructura institucional, recursos y gestión en el sistema escolar chileno. In: COX, C. (ed.). Políticas educacionales en el cambio de siglo: la reforma del sistema escolar en Chile. Santiago: Editorial Universitaria, 2003. 
JOFRÉ, G. Subvenciones en educación. Estudios Públicos, [s. 1.], n. 32, p. 31-55, 1988.

JOIKO, S. El cuasi-mercado educativo en Chile: desarrollo y consecuencias. Revista Electrónica Diálogos Educativos, Santiabo, v. 12, n. 23, p. 148-175, 2012.

LARRAÑAGA, O.; PEIRANO, C.; FALCK, D. El sector municipal en el sistema educacional chileno. In: MARCEL, M; RACZYNSKI, D. (ed.). La asignatura pendiente: claves para la revalidación de la educación pública de gestión local en Chile. Santiago: Uqbar Editores, 2009. p. 99-134.

MAINARDES, J. Abordagem do ciclo de políticas; uma contribucao para a análise de políticas educacionais. Educação e Sociedade, Campinas, v. 27, n. 94, p. 47-69, jan./abr. 2006. https://doi.org/10.1590/S0101-73302006000100003

MAYOL, A. Derrumbe del modelo: la crisis de la economía de mercado en el Chile contemporáneo. Santiago. LOM, 2012.

MOULIAN, T. Chile actual: anatomía de un mito. LOM: Santiago, 2002.

NÚÑEZ PRIETO, I. Educación chilena en la República: promesas de universalismo y realidades de inequidad en su historia. Psicoperspectivas, Valparaiso, v. 14, n. 3, p. 5-16, 2015. https://doi.org/10.5027/psicoperspectivas-Vol14-Issue3-fulltext-617

NÚÑEZ PRIETO, I. Las transformaciones educacionales bajo el Régimen Militar: programa interdisciplinario de investigaciones en educación, PIIE. Santiago: PIIE, 1984.

ORGANIZACIÓN PARA LA COOPERACIÓN Y EL DESARROLLO ECONÓMICO - OCDE. Chile: revisión de las políticas nacionales de educación. París, 2004.

ORGANIZACIÓN PARA LA COOPERACIÓN Y EL DESARROLLO ECONÓMICO - OCDE. Política educativa en perspectiva: hacer posible las reformas. Barcelona: Fundación Santillana; 2015

POLITEIA. Estudio mejoramiento de la gestión y la calidad de la gestión municipal. Informe ejecutivo por encargo de DIPLAP. Santiago: Ministerio de Educación, 2008. 
RACZYNSKI, D.; SALINAS, D. Prioridades, actores y procesos en la gestión municipal de la educación. In: MARCEL, M; RACZYNSKI, D. (eds.). La asignatura pendiente: claves para la revalidación de la educación pública de gestión local en Chile. Santiago: Uqbar, 2009. p. 135-176.

SABATIER, P. A. Top-down and bottom-up approaches to implementation research: a critical analysis and suggested synthesis. Journal of Public Policy, Ann Arbor, v. 6, n. 1, p. 21-48, jan./mar. 1986

SALVAT, P. Max Weber: poder y racionalidad: hacia una refundación normativa de la política. Santiago: Ril Ediciones, 2014.

SECCHI, L. Políticas públicas: conceitos, esquemas de análise, casos práticos 2. ed. Brasília, DF: Cengage Learning, 2014.

SEGOVIA, M. La deficiente ejecución de Ley de Nueva Educación Pública, el talón de Aquiles de la ministra Cubillos. el mostrador, Santiago, 30 sep. 2019. Disponible en: https://www.elmostrador.cl/noticias/pais/2019/09/30/ladeficiente-ejecucion-de-ley-de-nueva-educacion-publica-el-talon-de-aquilesde-la-ministra-cubillos/. Acceso en: 25 ago. 2019.

URIBE, M.; VALENZUELA, J. P.: ANDERSON, S. Estudio de seguimiento y sistematización de procesos críticos en la transición de la gestión de la educación pública de los municipios a los Servicios Locales de Educación. Santiago, Universidad de Chile, 2019.

VALDIVIA ORTIZ DE ZARATE, V. La "alcaldización de la política" en la post dictadura pinochetista: las comunas de Santiago, las condes y pudahuel. Izquierdas, Santiago, n. 38, p. 113-140, feb. 2018. https://doi.org/10.4067/S0718-50492018000100113

WOLLMANN, H. Policy evaluation and evaluation research. In: FISCHER, F.; MILLER, G. J.; SIDNEY, M. S. (orgs.). Handbook of public policy analysis: theory, politics, and methods. New York: Taylor \& Francis, 2007. p. 425-468.

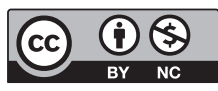

\section{Información del autor}

Sebastián Donoso-Díaz: Doctor en Educación, Universidad Academia de Humanismo Cristiano. Profesor Titular de la Universidad de Talca, Facultad de Ciencias de la Educación. Contacto: donoso.sebastian@gmail.com

iD https://orcid.org/0000-0002-4744-531X 